\title{
recillunds
}

Revista Cientifica Mundo de la Investigación y el Conocimiento

Zuleira Vanessa Torres Jaramillo a; David Arturo Paredes Bucheli ${ }^{\text {b; }}$ Andrés

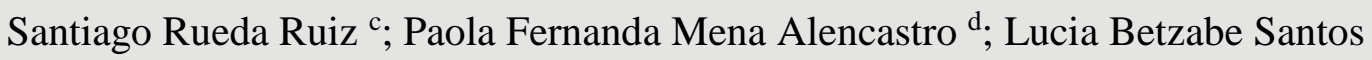

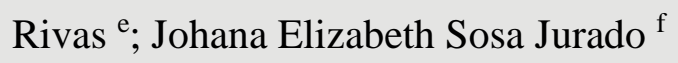

Tratamiento quirúrgico en pacientes pediátricos con problemas de hernia inguinal

Surgical treatment in pediatric patients with inguinal hernia problems

Revista Científica Mundo de la Investigación y el Conocimiento. Vol. 3 núm.2, abril, ISSN: 2588-073X, 2019, pp. 1248-1265

DOI: $10.26820 /$ recimundo/3.(2).abril.2019.1248-1265

URL: http://recimundo.com/index.php/es/article/view/499

Código UNESCO: 3205 Medicina Interna

Tipo de Investigación: Artículo de Investigación

\author{
Editorial Saberes del Conocimiento
}

Recibido: $15 / 01 / 2019$

Aceptado: 18/02/2019

Publicado: 30/04/2019

Correspondencia: zulev_2289@yahoo.com

\footnotetext{
a. Hospital General IESS; Neonatalogia; Médico Residente; Ibarra, Ecuador; zulev_2289@yahoo.com

b. Hospital de Especialidades Eugenio Espejo; Cirugía Cardíaca; Médico Residente; Quito, Ecuador; ddarthurp88@ gmail.com

c. Hospital General IESS; Servicio: Cirugía General; Médico Residente; Ambato, Ecuador; andres rr17@ hotmail.com

d. Centro de Salud Chimbacalle; Ministerio de Salud Pública; Servicio: Emergencia y Centro Obstétrico; Médico General; Quito, Ecuador; fher2814@gmail.com

e. Hospital Dr. Gustavo Domínguez Zambrano; Residente Servicio de Emergencia; Santo Domingo de los Tsachilas, Ecuador; lucybetsr@gmail.com

f. Hospital General Docente de Calderón; Pediatría; Médico Residente; Quito, Ecuador; joha_so21@ @hotmail.com
} 


\section{Tratamiento quirúrgico en pacientes pediátricos con problemas de hernia inguinal}

Vol. 3, núm. 2., (2019)

Zuleira Vanessa Torres Jaramillo; David Arturo Paredes Bucheli; Andrés Santiago Rueda Ruiz; Paola Fernanda Mena Alencastro; Lucia Betzabe Santos Rivas; Johana Elizabeth Sosa Jurado

\section{RESUMEN}

Se define la hernia como la protrusión de una parte de tejido u órgano a través de la pared que normalmente lo contiene. La incarceración es la complicación más grave de la hernia inguinal, se genera por la inflamación y edema gradual de una víscera atrapada en el orificio herniario. En pediatría es frecuente observar hernias inguinales indirectas, se producen porque el canal inguinal es más corto y cruza la pared abdominal en forma perpendicular, ocasionando que el anillo inguinal profundo y superficial se encuentre superpuestos. Se diagnostica por la información suministrada por los padres en la historia clínica y el examen físico. Según su ubicación anatómica la hernia puede ser: inguinal directa, inguinal indirecta, inguinal mixta, inguinoescrotal, crural o femoral e inguinal por deslizamiento. El tratamiento de la hernia inguinal en niños es quirúrgico, se debe realizar a fin de prevenir que el intestino quede atrapado y no pueda regresar al abdomen, ocasionando un elevado riesgo de perforación intestinal. Las técnicas quirúrgicas se pueden clasificar en tres grupos: técnicas abiertas en las que se podría emplear material protésico (-Lichtenstein) o no (Bassini, Shouldice) y técnicas laparoscópicas. Después de la cirugía de hernia inguinal en niños, se espera que los pacientes no padezcan de hernia o hinchazón escrotal. Cualquiera de los dos procedimientos abierto o laparoscópico son ambulatorios, con una noche de permanencia hospitalaria. Los pacientes reanudan su actividad cotidiana transcurridas 48 horas luego de la intervención. La metodología usada es descriptiva, con un enfoque documental, es decir, revisar fuentes disponibles en la red, como google académico, con contenido oportuno, actualizado y relevante desde el punto de vista científico que enriquezca el análisis del tema planteado en este artículo.

Palabras Claves: Hernia Inguinal; Cirugía; Recogimiento de Órganos con Mallas; Técnica Laparoscópica; Ambulatorio; Niños pre Término; Bulto; Protuberancia. 


\section{Tratamiento quirúrgico en pacientes pediátricos con problemas de hernia inguinal}

Vol. 3, núm. 2., (2019)

Zuleira Vanessa Torres Jaramillo; David Arturo Paredes Bucheli; Andrés Santiago Rueda Ruiz; Paola Fernanda Mena Alencastro; Lucia Betzabe Santos Rivas; Johana Elizabeth Sosa Jurado

\section{ABSTRACT}

The hernia is defined as the protrusion of a part of tissue or organ through the wall that normally contains it. Incarceration is the most serious complication of inguinal hernia, it is generated by the inflammation and gradual edema of a viscera trapped in the hernial orifice. In pediatric patients, it is common to see indirect inguinal hernias. They occur because the inguinal canal is shorter and crosses the abdominal wall perpendicularly, causing the deep and superficial inguinal ring to be superimposed. It is diagnosed by the information provided by the parents in the clinical history and the physical examination. According to its anatomical location the hernia can be: direct inguinal, indirect inguinal, mixed inguinal, inguinoscrotal, crural or femoral and inguinal by sliding. The treatment of inguinal hernia in children is surgical, should be performed in order to prevent the intestine is trapped and can not return to the abdomen, causing a high risk of intestinal perforation. Surgical techniques can be classified into three groups: open techniques in which prosthetic material could be used (Lichtenstein) or not (Bassini, Shouldice) and laparoscopic techniques. After inguinal hernia surgery in children, it is expected that patients do not suffer from hernia or scrotal swelling. Either open or laparoscopic procedure is ambulatory, with one night of hospital stay. The patients resume their daily activity after 48 hours after the intervention. The methodology used is descriptive, with a documentary approach, that is, to review sources available on the web, such as academic google, with timely, updated and relevant content from a scientific point of view that enriches the analysis of the topic raised in this article.

Key Words: Inguinal hernia; Surgery; Organ Collection with Meshes; Laparoscopic technique; Ambulatory; Pre-term children; Package; Boss. 


\section{Tratamiento quirúrgico en pacientes pediátricos con problemas de hernia inguinal}

Vol. 3, núm. 2., (2019)

Zuleira Vanessa Torres Jaramillo; David Arturo Paredes Bucheli; Andrés Santiago Rueda Ruiz; Paola Fernanda Mena Alencastro; Lucia Betzabe Santos Rivas; Johana Elizabeth Sosa Jurado

\section{Introducción.}

La hernia inguinal es la persistencia de un conducto que comunica el interior de la cavidad abdominal con el pubis (en las niñas) y con la bolsa escrotal (en niños). Tiene el aspecto de un bulto o protuberancia en la ingle o escroto. Esa protuberancia se hace evidente cuando el niño llora, tose o se pone de pie, cuando el niño se acuesta ese bulto tiende a desaparecer. Son más comunes en varones que en hembras.

La hernia inguinal se manifiesta en forma de bulto que aparece y desaparece en la zona inguinal. En los niños puede llegar a ocupar el escroto y ser de un tamaño grande mientras que en las niñas se manifiesta como un pequeño nódulo (equivalente a una aceituna) móvil y no doloroso que correspondería al ovario. Si el niño presenta una protuberancia fija, dura, muy dolorosa, un malestar general, vómitos e irritabilidad, es conveniente acudir a urgencias a un pediatra.

Mediante la evaluación física del niño y la información aportada por los padres, el pediatra diagnosticará si se trata de una hernia inguinal u otra patología. El tratamiento de la hernia inguinal es quirúrgico cuya técnica la definirán el cirujano pediátrico y sus padres, lo importante a considerar es minimizar el riesgo que el intestino quede atrapado y no pueda regresar al abdomen.

El contenido a desarrollar en el presente artículo es explicar el proceso de diagnóstico de la hernia inguinal y diferenciar sus tipos, como técnicas quirúrgicas que no solo aliviaran al niño sino que pueden prevenir daños graves en materia de salud al infante si no es tratado a tiempo. 


\section{Tratamiento quirúrgico en pacientes pediátricos con problemas de hernia inguinal}

Vol. 3, núm. 2., (2019)

Zuleira Vanessa Torres Jaramillo; David Arturo Paredes Bucheli; Andrés Santiago Rueda Ruiz; Paola Fernanda Mena Alencastro; Lucia Betzabe Santos Rivas; Johana Elizabeth Sosa Jurado

La gama de técnicas y opciones de tratamiento también reflejan la disponibilidad que en materia de médicos y experiencia ofrece ese país, su costo y nivel de vida de esa población.

\section{Metodología.}

Esta investigación está dirigida al estudio "Tratamiento quirúrgico en pacientes pediátricos con problemas de hernia inguinal". Para realizarlo se usó una metodología descriptiva, con un enfoque documental, es decir, revisar fuentes disponibles en la red, como google académico, con contenido oportuno y relevante desde el punto de vista científico para dar respuesta a lo tratado en el presente artículo y que sirvan de inspiración para realizar otros proyectos. Las fuentes consultadas pueden ser estudiadas al final, en la bibliografía.

\section{Resultados.}

Las hernias inguinales en los niños ocurren con más frecuencia cuando la abertura de la ingle presente en el feto no se cierra por completo al nacer. (Boston Children's Hospital, 2016) Esta abertura está presente en todo niño para permitir que los testículos bajen hasta el escroto. El contenido del abdomen puede pasar a través de esa abertura.

Algunos factores que aumentan la probabilidad de desarrollar hernias inguinales en niños:

Niños prematuros.

Testículos que no bajen.

Antecedentes familiares de hernias. 


\section{Tratamiento quirúrgico en pacientes pediátricos con problemas de hernia inguinal}

Vol. 3, núm. 2., (2019)

Zuleira Vanessa Torres Jaramillo; David Arturo Paredes Bucheli; Andrés Santiago Rueda Ruiz; Paola Fernanda Mena Alencastro; Lucia Betzabe Santos Rivas; Johana Elizabeth Sosa Jurado

Fibrosis quísticas.

Displasia del desarrollo de cadera.

Anormalidades de la uretra.

Se define la hernia como la protrusión de una parte de tejido u órgano a través de la pared que normalmente lo contiene. La incarceración es la complicación más grave de la hernia inguinal, se genera por la inflamación y edema gradual de una víscera atrapada en el orificio herniario. Al incrementarse la presión, se compromete el drenaje venoso y linfático, el flujo de entrada arterial disminuye y aparece secuencialmente infarto, necrosis y gangrena, esta situación requerirá intervención quirúrgica urgente (Gutiérrez, 2001).

En pediatría es frecuente observar hernias inguinales indirectas, se producen porque el canal inguinal es más corto y cruza la pared abdominal en forma perpendicular, ocasionando que el anillo inguinal profundo y superficial se encuentre superpuestos. Se diagnostica por la información suministrada por los padres en la historia clínica y el examen físico.

Son comunes en los niños, más del $90 \%$ de las hernias inguinales son diagnosticadas en varones, un $60 \%$ afecta el lado derecho, $25 \%$ sólo el lado izquierdo y un $15 \%$ se manifiesta en forma bilateral. Las estadísticas así lo acreditan (Villarroel, 2017):

$1-5 \%$ de los recién nacidos a término.

$9-11 \%$ de los recién nacidos pre términos. 


\section{Tratamiento quirúrgico en pacientes pediátricos con problemas de hernia inguinal}

Vol. 3, núm. 2., (2019)

Zuleira Vanessa Torres Jaramillo; David Arturo Paredes Bucheli; Andrés Santiago Rueda Ruiz; Paola Fernanda Mena Alencastro; Lucia Betzabe Santos Rivas; Johana Elizabeth Sosa Jurado

$100 \%$ de los recién nacidos pre términos cuyo peso es menor de 900 grs.

Al acudir a consulta, los padres manifiestan observar la aparición de un bulto en la zona inguinal del niño, puede extenderse hacia el escroto o vulva. Esta protuberancia puede cambiar de tamaño debido al llanto, al defecar, etc. Mediante el examen físico se revisa desde el abdomen inferior hasta el escroto o vulva, se buscará presencia de bultos o asimetrías en el trayecto del canal inguinal, búsqueda de anomalías genitales.

La palpitación puede realizarse de pie y en supino para aprovechar el aumento de la presión intraabdominal, otra técnica es solicitar al niño que realice maniobras de valsaba y posteriormente realizar la palpación. La tabla $\mathrm{N}^{\circ} 1$ mostrada a continuación, resume los métodos de diagnósticos más usados.

\section{Tabla $N^{\circ}$ 1. Diagnósticos diferenciales de hernia inguinal en niños}

\begin{tabular}{|l|l|}
\hline Patología & Aspectos a observar por el médico tratante \\
\hline Criptorquidia & $\begin{array}{l}\text { Bulto a nivel del canal inguinal, no se palpa el } \\
\text { testículo en escroto y puede observarse un } \\
\text { escroto hipoplásico. }\end{array}$ \\
\hline Hidrocele & $\begin{array}{l}\text { Aumento del volumen inguinoescrotal, el examen } \\
\text { físico muestra una transiluminación positiva. }\end{array}$ \\
\hline Linfadenopatía inguinal & $\begin{array}{l}\text { A diferencia de la hernia inguinal es precedido } \\
\text { por una infección genital o de extremidades } \\
\text { inferiores. }\end{array}$ \\
\hline Tumor testicular & $\begin{array}{l}\text { Es un bulto testicular de consistencia pétrea que } \\
\text { no cambia con maniobras de valsalva. }\end{array}$ \\
\hline Varicocele & $\begin{array}{l}\text { Masa tortuosa donde destacan las venas } \\
\text { varicosas, puede haber dolor o sensación de } \\
\text { pesadez al estar de pie y disminuye al acostarse. }\end{array}$ \\
\hline
\end{tabular}




\section{Tratamiento quirúrgico en pacientes pediátricos con problemas de hernia inguinal}

Vol. 3, núm. 2., (2019)

Zuleira Vanessa Torres Jaramillo; David Arturo Paredes Bucheli; Andrés Santiago Rueda Ruiz; Paola Fernanda Mena Alencastro; Lucia Betzabe Santos Rivas; Johana Elizabeth Sosa Jurado

Fuente: Villarroel (2017)

La criptorquidia es una localización anómala del testículo fuera de la bolsa escrotal, entre la cavidad abdominal y la bolsa intra-abdominal, inguinal. Su etiología es un defecto embrionario entre el $6-7$ mes. La localización más frecuente es el conducto inguinal. En caso de diagnóstico dudoso mediante el tacto (niños obesos), la ecografía resulta de utilidad. Tiene indicación quirúrgica siempre que se asocie a una hernia inguinal o a partir del primer año de vida si no desciende espontáneamente antes de los 18 -24 meses de vida. Es más urgente si el teste no es palpable ni resulta ecográficamente localizable en conducto inguinal (Martínez, 2008).

La hidrocele es la acumulación de líquido alrededor del testículo (vaginal testicular). La habitual en el neonato es la del tipo indirecto por persistencia de un conducto perito neo vaginal permeable. La de tipo directo (debilidad del piso del conducto inguinal) son excepcionales en el recién nacido. La fisiopatología en el caso de la hernia inguinal es un orificio grande, que permite la protrusión de intestino delgado. Su tratamiento es operación tanto en neonatos y prematuros para prevenir lesiones testiculares en los niños y de un ovario o de una trompa en las niñas.

Según su ubicación anatómica la hernia puede ser (P.Gac, 2001):

Inguinal directa: la hernia emerge por dentro de los vasos epigástricos conformando un defecto dependiente de la pared posterior del conducto. 


\section{Tratamiento quirúrgico en pacientes pediátricos con problemas de hernia inguinal}

Vol. 3, núm. 2., (2019)

Zuleira Vanessa Torres Jaramillo; David Arturo Paredes Bucheli; Andrés Santiago Rueda Ruiz; Paola Fernanda Mena Alencastro; Lucia Betzabe Santos Rivas; Johana Elizabeth Sosa Jurado

Inguinal indirecta: la hernia emerge fuera de los vasos epigástricos aprovechando la debilidad en el orificio inguinal profundo, por su ubicación el saco herniario quedan envueltos por el cremáster, en contacto con el cordón espermaticida.

Inguinal mixta: coexiste una hernia inguinal directa con una indirecta.

Inguinoescrotal: es una hernia indirecta que por su tamaño alcanza al escroto, pudiendo ocuparlo por completo hasta dejarlo a tensión.

Crural o femoral: existe un defecto en el anillo crural por donde florece el saco herniario. Es más frecuente en mujeres.

Inguinal por deslizamiento: dentro del saco herniario se encuentra una víscera que se deslizó junto con el saco, como contenido deslizado se tiene: vejiga, colón, sigmoides o ciego.

\section{Tratamiento}

El tratamiento de la hernia inguinal en niños es quirúrgico, se debe realizar a fin de prevenir que el intestino quede atrapado y no pueda regresar al abdomen, ocasionando un elevado riesgo de perforación intestinal. Si por el contrario, es el ovario lo que está fuera y no regresa a su posición, el paciente estaría expuesto a traumatismo y riesgo de torsión. En la intervención se separa el conducto herniario (saco) del resto del tejido vecino, se corta y se cierra con una sutura. Es poco probable colocar mallas en los niños, solamente en casos extremos combinados con otras patologías. La intervención se hace con anestesia general. 


\section{Tratamiento quirúrgico en pacientes pediátricos con problemas de hernia}

inguinal

Vol. 3, núm. 2., (2019)

Zuleira Vanessa Torres Jaramillo; David Arturo Paredes Bucheli; Andrés Santiago Rueda Ruiz; Paola Fernanda Mena Alencastro; Lucia Betzabe Santos Rivas; Johana Elizabeth Sosa Jurado

Las técnicas quirúrgicas se pueden clasificar en tres grupos: técnicas abiertas en las que se podría emplear material protésico (Lichtenstein, Rutkow-Robbins, etc.) o no (Bassini, Shouldice, McVay, etc.) y técnicas laparoscópicas protésica.

Técnica de Lichtenstein: es ideal en la reparación de la hernia inguinal unilateral o bilateral primarias o recurrentes, si la primera intervención se efectuó por laparoscópica. Menos apropiada para hernias inguinales con grandes orificios herniarios en cuyo caso se aconsejaría la técnica Rutkow-Robbins. Es una técnica rápida y sencilla, permite tratar procesos ambulatorios en forma segura, minimizando el riesgo de complicaciones precoces, sin drenajes y control del dolor por vía oral (Turiño, 2018).

La malla es un material de prótesis no absorbible en forma de red que se coloca para refuerzo de los músculos de la zona donde se ubica la hernia. Existen diferentes tipos de materiales, formas de mallas, la más usada es la malla de polipropileno, su uso reduce significativamente la tasa de recaída de las hernioplastías inguinales de 11\% a 1-2\% (Sadava, 2016)

Dentro de sus ventajas está la ausencia de tensión en reparación del defecto, mejora el dolor y disminuye el riesgo de recurrencia. Las nuevas mallas de bajo peso molecular y autofijables, disminuyen el riesgo de neuropatía por atrapamiento al no requerir sutura para su anclaje a las estructuras anatómicas.

Técnica Bassini: es una reparación de tensión en el que los bordes del defecto se vuelven a coser sin usar ningún tipo de malla. El tendón conjunto formado por los extremos distales de 


\section{Tratamiento quirúrgico en pacientes pediátricos con problemas de hernia inguinal}

Vol. 3, núm. 2., (2019)

Zuleira Vanessa Torres Jaramillo; David Arturo Paredes Bucheli; Andrés Santiago Rueda Ruiz; Paola Fernanda Mena Alencastro; Lucia Betzabe Santos Rivas; Johana Elizabeth Sosa Jurado

los transversos del abdomen y por los músculos oblicuos internos, se aproximan al ligamento inguinal y se cierra.

Técnica de Shouldice: es la técnica principal basada en la sutura. Se reconstruye cuatro capas de la fascia transversallis relativamente complicadas. En manos del cirujano experto en esta técnica, se logra obtener bajas tasas de recurrencia. Es más doloroso el post operatorio que con las otras técnicas vistas anteriormente.

La principal ventaja de la técnica de Shouldice es el dolor crónico bajo en comparación con la reparación de hernia inguinal por el método de malla Lichtenstein, similar al generando por una intervención de Laparoscopia. En países donde adquirir las mallas resulte muy costoso, la técnica de Shouldice es oportuna siempre que existan cirujanos altamente capacitados y efectivos en ese tipo de intervención.

Otra ventaja de las reparaciones de hernias inguinales basado en la sutura frente a las reparaciones de malla permanente es la no introducción en el cuerpo de un material extraño o de polipropileno, pudiendo ocasionar complicaciones a largo plazo.

La herniorrafia por vía inguinal es una de las intervenciones frecuentemente realizada por los cirujanos pediátricos y el abordaje por vía laparoscópica. Entre las ventajas que ofrece la reparación laparoscópica versus la cirugía abierta tenemos: el cierre de defectos bilaterales sin incisiones adicionales, mejor visualización de las estructuras del cordón, menor tiempo operatorio en los defectos bilaterales, disminución del dolor post operatorio y una incorporación más rápida a las actividades cotidianas. (Fuente, 2018). 


\section{Tratamiento quirúrgico en pacientes pediátricos con problemas de hernia}

inguinal

Vol. 3, núm. 2., (2019)

Zuleira Vanessa Torres Jaramillo; David Arturo Paredes Bucheli; Andrés Santiago Rueda Ruiz; Paola Fernanda Mena Alencastro; Lucia Betzabe Santos Rivas; Johana Elizabeth Sosa Jurado

Técnicas laparoscópicas: El abordaje laparoscópico se puede realizar transperitoneal o preperitoneal con visualización transperitoneal.

Perperitoneal: un pequeño gancho cargado con una sutura se pasa alrededor del anillo profunda posterior a la incisión en la piel inguinal. El paso de la sutura se observa mediante un endoscopio en el obligo, la ligadura se lleva a continuación extra corporal y atada, cerrando el orificio herniario.

Un solo trocar o transperitoneal: se usa un solo trocar umbilical para la introducción de una óptica que controlará intra abdominalmente el cierre transcutáneo del orificio inguinal. La técnica subcutánea con aguja SEAL, el cierre del anillo se realiza con una aguja de sutura curva introducida a través de una incisión inguinal mínima. (Molina, 2012)Años más tarde se desarrolló un nuevo método percutáneo con aguja vascular (PIRS) mediante el cual se rodea el anillo, permitiendo un mínimo gap a nivel de los vasos y deferente.

Las posibles desventajas de esta técnica son:

Posible aumento en el tiempo operatorio en los defectos unilaterales.

Elevado costo.

Requerimiento de intubación oro traqueal para la anestesia.

Experiencia comprobada del médico en esta técnica. 


\section{Tratamiento quirúrgico en pacientes pediátricos con problemas de hernia inguinal}

Vol. 3, núm. 2., (2019)

Zuleira Vanessa Torres Jaramillo; David Arturo Paredes Bucheli; Andrés Santiago Rueda Ruiz; Paola Fernanda Mena Alencastro; Lucia Betzabe Santos Rivas; Johana Elizabeth Sosa Jurado

Entre las complicaciones más frecuentes de la herniorrafia inguinal se observan: la hidrocele, la infección de la herida quirúrgica, el edema escrotal, eritema, la atrofia testicular, el ascenso testicular y la recidiva. La técnica laparoscópica presenta complicaciones propias con respecto a la técnica abierta como la hipercapnia, invasión intraperitoneal y la lesión visceral o vascular ocasionado por los tocares e instrumentos utilizados.

La mayoría de las pruebas en apoyo de resultados empíricos para una hernia inguinal de cual tratamiento es el más idóneo, depende del tipo de hernia, la información obtenida mediante una exploración bilateral. La evaluación laparoscópica umbilical detecta entre un 30 - 50\% de los pacientes y es un hallazgo similar a la de la cirugía abierta contralateral. No hay aumento significativo en la morbilidad del tratamiento rutinario de hernia inguinal por cualquiera de las técnicas anteriormente nombradas (Internacional Pediatric Endosurgery Group, 2009).

La reparación temprana antes de la descarga se justifica en pacientes con edad gestacional por debajo de las 50 semanas y/o con antecedentes de encarcelamiento. Estudios revelan que la reparación de la hernia inguinal laparoscópica transperitoneal tiene una ventaja sobre el procedimiento abierto quirúrgico en la capacidad de detectar y reparar simultáneamente. La tasa de recurrencia después de la reparación laparoscópica también es superior y está directamente proporcional con la experiencia de cirujano.

Después de la cirugía de hernia inguinal en niños se espera que los pacientes no padezcan de hernia o hinchazón escrotal. Cualquiera de los dos procedimientos abierto o laparoscópico son ambulatorios, con una noche de permanencia hospitalaria en pacientes con edad comprendida entre las 44 - 50 semanas de gestación (Internacional Pediatric Endosurgery Group, 2009). La 


\section{Tratamiento quirúrgico en pacientes pediátricos con problemas de hernia}

inguinal

Vol. 3, núm. 2., (2019)

Zuleira Vanessa Torres Jaramillo; David Arturo Paredes Bucheli; Andrés Santiago Rueda Ruiz; Paola Fernanda Mena Alencastro; Lucia Betzabe Santos Rivas; Johana Elizabeth Sosa Jurado

incidencia de desarrollar una hernia metacrónica posterior a la hernia inguinal es de 6 a 8\%. Los pacientes reanudan su actividad cotidiana transcurridas 48 horas luego de la intervención.

Complicaciones a corto plazo posteriores a la operación son poco probable, lesión en el conducto deferente durante la reparación inguinal es un riesgo latente. La recurrencia después de la intervención de la hernia inguinal está relacionada con factores:

La prematuridad.

Aumento de la presión abdominal.

Infección y hematoma de la herida post operatoria.

Experiencia del cirujano pediátrico.

En forma de resumen se muestra en la Tabla $\mathrm{N}^{\circ} 2$ las ventajas y desventajas de la cirugía laparoscópica versus la cirugía abierta, la decisión final la toman el médico tratante y la familia del paciente.

\section{Tabla $N^{\circ}$ 2. Laparoscopia versus Cirugía abierta}

\begin{tabular}{|c|c|}
\hline Ventajas & Desventajas \\
\hline $\begin{array}{l}\text { En laparoscopia la recuperación es más } \\
\text { rápida que el otro procedimiento. } \\
\text { Menos dolor durante los primeros días } \\
\text { posteriores a la intervención. } \\
\text { Menos complicaciones post operatorias } \\
\text { como infecciones y hemorragias. }\end{array}$ & $\begin{array}{l}\text { Requerimiento de cirujano con } \\
\text { comprobada experiencia en técnica } \\
\text { laparoscópica. } \\
\text { Una mala intervención laparoscópica } \\
\text { acarrea recurrencia de la hernia primaria. } \\
\text { El costo de la técnica laparoscópica es } \\
\text { mayor que en la abierta. }\end{array}$ \\
\hline
\end{tabular}

Fuente: (Sadava, 2016) 


\section{Tratamiento quirúrgico en pacientes pediátricos con problemas de hernia inguinal}

Vol. 3, núm. 2., (2019)

Zuleira Vanessa Torres Jaramillo; David Arturo Paredes Bucheli; Andrés Santiago Rueda Ruiz; Paola Fernanda Mena Alencastro; Lucia Betzabe Santos Rivas; Johana Elizabeth Sosa Jurado

El tomar o rechazar un procedimiento quirúrgico en relación a la variable costo en este tipo de intervención es relativo, es cierto que la cirugía laparoscópica es más costosa que la cirugía abierta pero la recuperación más rápida del paciente mediante esta técnica, abarata su costo a la hora de elegir un método considerando la decisión en la variable económica.

\section{Conclusiones.}

Una hernia inguinal se produce por una abertura o debilidad de la pared del musculo abdominal inferior, parte del contenido del abdomen como los intestinos puede pasar a través de esa abertura. Son comunes en bebes y niños. Algunos factores que potencias el padecimiento de hernia inguinal son:

Niños prematuros.

Testículos que no han bajado.

Antecedentes familiares de hernia.

Fibrosis quísticas.

Displasia del desarrollo de la cadera.

Anormalidades de la uretra.

En pediatría es frecuente observar hernias inguinales indirectas, se producen porque el canal inguinal es más corto y cruza la pared abdominal en forma perpendicular, ocasionando que el anillo inguinal profundo y superficial se encuentre superpuestos. Se diagnostica por la 


\section{Tratamiento quirúrgico en pacientes pediátricos con problemas de hernia}

inguinal

Vol. 3, núm. 2., (2019)

Zuleira Vanessa Torres Jaramillo; David Arturo Paredes Bucheli; Andrés Santiago Rueda Ruiz; Paola Fernanda Mena Alencastro; Lucia Betzabe Santos Rivas; Johana Elizabeth Sosa Jurado

información suministrada por los padres en la historia clínica y el examen físico. Al acudir a consulta, los padres manifiestan observar la aparición de un bulto en la zona inguinal del niño, puede extenderse hacia el escroto o vulva. Esta protuberancia puede cambiar de tamaño debido al llanto, al defecar, etc. Mediante el examen físico se revisa desde el abdomen inferior hasta el escroto o vulva, se buscará presencia de bultos o asimetrías en el trayecto del canal inguinal, búsqueda de anomalías genitales.

El tratamiento de la hernia inguinal en niños es quirúrgico, se debe realizar a fin de prevenir que el intestino quede atrapado y no pueda regresar al abdomen, ocasionando un elevado riesgo de perforación intestinal. Si por el contrario, es el ovario lo que está fuera y no regresa a su posición, el paciente estaría expuesto a traumatismo y riesgo de torsión. En la intervención se separa el conducto herniario (saco) del resto del tejido vecino, se corta y se cierra con una sutura. Es poco probable colocar mallas en los niños, solamente en casos extremos combinados con otras patologías. La intervención se hace con anestesia general.

Las técnicas quirúrgicas se pueden clasificar en tres grupos: técnicas abiertas en las que se podría emplear material protésico Lichtenstein (es ideal en la reparación de la hernia inguinal unilateral o bilateral primarias o recurrentes, es una técnica rápida y sencilla, permite tratar procesos ambulatorios en forma segura, minimizando el riesgo de complicaciones precoces, sin drenajes y control del dolor por vía oral. La malla es un material de prótesis no absorbible en forma de red que se coloca para refuerzo de los músculos de la zona donde se ubica la hernia), Bassini (es una reparación de tensión en el que los bordes del defecto se vuelven a coser sin usar ningún tipo de malla), Shouldice (es la técnica principal basada en la sutura, es más doloroso el 


\section{Tratamiento quirúrgico en pacientes pediátricos con problemas de hernia inguinal}

Vol. 3, núm. 2., (2019)

Zuleira Vanessa Torres Jaramillo; David Arturo Paredes Bucheli; Andrés Santiago Rueda Ruiz; Paola Fernanda Mena Alencastro; Lucia Betzabe Santos Rivas; Johana Elizabeth Sosa Jurado

post operatorio que con las otras técnicas vistas anteriormente y la principal ventaja de la técnica de Shouldice es el dolor crónico bajo en comparación con la reparación de hernia inguinal por el método de malla Lichtenstein).

La técnicas laparoscópica se usa un solo trocar umbilical para la introducción de una óptica que controlará intraabdominalmente el cierre transcutáneo del orificio inguinal. La técnica subcutánea con aguja SEAL, el cierre del anillo se realiza con una aguja de sutura curva introducida a través de una incisión inguinal mínima.

Estudios revelan que la reparación de la hernia inguinal laparoscópica transperitoneal tiene una ventaja sobre el procedimiento abierto quirúrgico en la capacidad de detectar y reparar simultáneamente. La tasa de recurrencia después de la reparación laparoscópica también es superior y está directamente proporcional con la experiencia de cirujano. El tomar o rechazar un procedimiento quirúrgico en relación a la variable costo en este tipo de intervención es relativo, es cierto que la cirugía laparoscópica es más costosa que la cirugía abierta pero la recuperación más rápida del paciente mediante esta técnica, abarata su costo a la hora de elegir un método considerando la decisión en la variable coste-beneficio.

\section{Bibliografía.}

Boston Children's Hospital. (15 de Julio de 2016). Hernias inguinales en niños. Obtenido de Family Education Sheet.: www.childrenhospital.org

Fuente, L. D. (2018). Reparación laparoscópica de la hernia inguinal e hidrocele en edad pediátrica. Revista Cirugía Pediátrica, Volumen 31. N³, 125-129.

Gutiérrez, J. (2001). Urgencias quirúrgicas en niños. Boletín de la Sociedad de Pediatría de Asturias, $N^{\circ} 41,91-98$. 


\section{Tratamiento quirúrgico en pacientes pediátricos con problemas de hernia inguinal}

Vol. 3, núm. 2., (2019)

Zuleira Vanessa Torres Jaramillo; David Arturo Paredes Bucheli; Andrés Santiago Rueda Ruiz; Paola Fernanda Mena Alencastro; Lucia Betzabe Santos Rivas; Johana Elizabeth Sosa Jurado

Internacional Pediatric Endosurgery Group. (4 de Noviembre de 2009). Directrices IPEG de hernia inguinal e hidrocele. Obtenido de IPEG: www.ipeg.org

Martínez, C. (2008). Urgencias quirúrgicas urogenotales. Asociación Española de Pediatría, 476-491.

Molina, M. (2012). Tratamiento percutáneo asistido por laparoscopia de la hernia inguinal infantil. Revista Cirugía Pediátrica, Volumen 25, $N^{\circ} 1,4-8$.

P.Gac. (2001). Hernia inguinal: un desafio quirúrgico permanente. Artículo Docente, Universidad Austral de Chile, 96-106.

Sadava, E. (2016). Hernia inguinal. Ventaja de la cirugía laparoscópica. Obtenido de www.hospitalaleman.org.ar

Turiño, J. (2018). Hernioplastia inguinal abierta: Técnica de Lichtenstein. Cirugía Andaluz, Volumen $29 N^{\circ} 2,160-162$.

Villarroel, M. (2017). Hernia inguinal en niños: Evaluación y diagnósticos diferenciales. Obtenido de www.medicina.uc.cl 